\title{
Response Stratification in the First-Line Combined Immunotherapy of Hepatocellular Carcinoma at Genomic, Transcriptional and Immune Repertoire Levels
}

\author{
Jiamin Cheng ${ }^{1, *}$ \\ Yinyin $\mathrm{Li}^{1, *}$ \\ Xiaohui Wang ${ }^{2, *}$ \\ Zheng Dong' \\ Yan Chen' \\ Rui Zhang' \\ Jiagan Huang' \\ Xueyuan Jin \\ Jianfei $\mathrm{Yao}^{2}$ \\ Aifang $\mathrm{Ge}^{2}$ \\ Lele Song $\mathbb{1 D}^{2,3, *}$ \\ Yinying Lu' \\ Zhen Zeng' \\ 'Comprehensive Liver Cancer \\ Department, The Fifth Medical Center of \\ the Chinese PLA General Hospital, \\ Beijing, 100039, People's Republic of \\ China; ${ }^{2}$ HaploX Biotechnology, Shenzhen, \\ Guangdong Province, People's Republic \\ of China; ${ }^{3}$ Department of Radiotherapy, \\ The Eighth Medical Center of the \\ Chinese PLA General Hospital, Beijing, \\ I0009I, People's Republic of China
}

*These authors contributed equally to this work
Correspondence: Yinying Lu; Zhen Zeng Comprehensive Liver Cancer Department, The Fifth Medical Center of the Chinese PLA General Hospital, Beijing, 100039, People's Republic of China

Email luyinying1973@163.com;

zengzhen1970@sina.com
Background: Immunotherapy combined with VEGF inhibitor has become the new first-line therapy for advanced or metastatic hepatocellular carcinoma (HCC). However, the biomarkers for response and prognosis stratification of HCC first-line combined immunotherapy have not been clarified.

Methods: Here, we obtained the genomic alteration data from pre-therapeutic samples of 103 HCC patients using a 605-gene NGS test, and obtained the transcriptional and T cell receptor (TCR) diversity data from 18 patients who underwent the first-line combined immunotherapy using RNAseq and TCR sequencing, respectively. Patients received sorafenib/sintilimab or lenvatinib/sintilimab combined first-line therapy and the response was assessed at 3-6 cycles of therapy. Results: No stratification of response was found in high-frequency key driver gene mutations, including TP53 and TERT. However, significantly higher ratio of progression (PD) was found in patients carrying MDM4 amplification. Similarly, FGF/3/4/19 amplifications could also result in high ratio of PD. The mRNA and lncRNA levels of eight genes related to hepatic metabolism and immune microenvironment exhibited significant differences between PR/SD and PD group, including DGKI, TNFSF14, CHST4, ACTIN1, PFKP, SLC51B, LCK and ERN1, suggesting stratification of response. Furthermore, moderate correlation was identified between the stratification genes (CHST4, SLC51B and ERN1) and immune factors (TIGIT, CD34, ICAM1, CCL5, CXCL9 and CXCL10), suggesting potential roles of these factors in immunoregulation. Strong linear correlation was found between any two of the three indexes for TCR CDR3 diversity, including Shannon-Wiener Index, Simpson index and evenness. However, no significant difference was found in the three indexes between the $\mathrm{PR} / \mathrm{SD}$ and PD group, suggesting no stratification of response by these indexes.

Conclusion: We identified several potential biomarkers for response stratification in the first-line combined immunotherapy. MDM4 was capable of predicting disease progression, and a panel mRNA and lncRNA of eight genes may also predict the response. Further validation is needed to verify these biomarkers.

Keywords: hepatocellular carcinoma, HCC, immunotherapy, mRNA, lncRNA, TCR, sintilimab, sorafenib, lenvatinib

\section{Introduction}

Hepatocellular carcinoma (HCC) is one of the leading causes of tumour-related death worldwide. The largest attributable causes for $\mathrm{HCC}$ are chronic infection by hepatitis $\mathrm{B}$ virus (HBV) and hepatitis $\mathrm{C}$ virus $(\mathrm{HCV}) .{ }^{1,2}$ Despite great advances in the diagnosis 
and treatment of HCC, the late-stage presentation is due in a large part to the absence of symptoms in early stages of the disease. Potentially curative options such as surgical resection are only indicated for early stage disease and are possible in only $10 \%$ to $20 \%$ of HCC patients, while the majority of patients with advanced $\mathrm{HCC}$ are not amenable to surgery with a poor prognosis. ${ }^{3}$ As a systemic treatment, immunotherapy has made great progress and attached much attention in HCC therapy. ${ }^{4,5}$ It is of great significance to search for new biomarkers related to HCC immunotherapy in order to further improve the efficacy, especially when first-line HCC immunotherapy has become an effective option.

Immunotherapy at multiple lines of therapy of HCC has been investigated. In Checkmate040 study, Nivolumab was used as the 2nd line treatment for advanced HCC patients who received the first-line sorafenib treatment. The objective response rate (ORR) of patients with PDL1 $\geq 1 \%$ was $26 \%$, compared with $19 \%$ for those with PDL1 $<1 \%{ }^{6}$ In Checkmate459 study, Nivolumab was compared with sorafenib as the first-line treatment. The results showed that the ORR was $28 \%$ for patients with PD-L1 $\geq 1$ $\%$, compared with $12 \%$ for those with PD-L1<1\%. Both studies suggested that patients with high PD-L1 expression had higher ORR in immunotherapy. ${ }^{7}$ Similarly, Keynote224 study and the Phase III Keynote240 study also showed benefit from pembrolizumab in patients with high PD-L1 expression. ${ }^{8,9}$ More recently, IMbravel50 study demonstrated that atezolizumab combined with bevacizumab was superior to the existing standard therapy of sorafenib at first-line therapy for HCC. ${ }^{10}$ In addition, phase IB study with pembrolizumab/lenvatinib combination at first-line and Phase II study with Carrelizumab/ FOLFOX4/GEMOX combination at first-line treatment of advanced HCC have shown better safety and efficacy than immunotherapy or chemotherapy alone. ${ }^{11,12}$ These results indicate that first-line combined immunotherapy for HCC may provide better response than second-line treatment and first-line single-drug treatment. However, the potential biomarkers for the first-line combined immunotherapy have not investigated, and the molecular mechanism for the first-line combined immunotherapy also needs to be clarified. In this study, we conducted comprehensive studies at genomic, transcriptional and immune repertoire levels to explore the relationship between the response of first-line combined immunotherapy and molecular biomarkers. We expect to provide helpful information for the stratification and prediction of clinical efficacy and prognosis of the first-line combined immunotherapy.

\section{Methods and Materials Ethics Approval and Consent to Participate}

All experiment plans and protocols for the study were submitted to the ethics/licensing committee of the participating hospitals for review and approval before the start of the clinical study, and were approved by the corresponding committee of the hospital. All subjects signed the informed consent before tissue or blood collection, and they were informed of the usage of samples and the test results. Informed consent was collected and obtained from all subjects before the start of the clinical study. All experiments, methods, procedures and personnel training were carried out in accordance with relevant guidelines and regulations of the participating hospitals and laboratory. This study was conducted in accordance with the Declaration of Helsinki.

\section{Study Design, Patients and Samples}

A prospective cohort study was designed and implemented to include as many intermediate or advanced stage HCC patients as possible (BCLC stage B and C), as long as the tissue or blood samples were available for NGS. The main inclusion criteria included: 1) adults over 18 years old and those have complete clinicopathological information and confirmed diagnosis of HCC by pathological examination; 2) BCLC-B or BCLC-C patients who do not have surgery opportunity and are suitable for first-line combined immunotherapy; 3) patients who plan to receive therapy in our hospital and available for follow-up; 4) patients have not received any systemic therapy before tissue or blood samples were collected. The main exclusion criteria included those with no tissue samples or control samples available. Patients with incomplete information or loss of follow-up were also excluded. As a result, a total of 103 patients with BCLC-B or BCLC-C HCC were recruited in this study. Clinical status of patients was determined before the collection of samples, including fresh surgical samples, needle biopsy samples, formalin-fix paraffin-embedded (FFPE) samples or blood that were obtained from all subjects who were confirmed with diagnosis of HCC. FFPE or fresh tissue samples were obtained for genomic test by NGS, using the blood peripheral lymphocytes as the control. Fresh cancer tissue samples and adjacent normal tissue samples were used 
for mRNA and lncRNA sequencing (RNA-seq), and blood samples were used for $\mathrm{T}$ cell repertoire sequencing (TCRseq). Samples with tumor cell content $\geq 20 \%$ were used for subsequent sequencing, while patients with tumor cell content less than $20 \%$ were asked to resample. In the 103 patients who had samples available for 605-gene panelbased genomic analysis, 78 patients were excluded due to surgical treatment or willing to receive therapy at local hospitals, 25 patients were available for the first-line combined immunotherapy in our hospital (Table 1, Supplementary Figure 1), in which 18 patients had paired cancer and adjacent normal samples for RNA-seq/TCR-seq and complete response data from the first-line combined immunotherapy. Data of RNA-seq and TCR-seq were obtained from 17 patients, as the sample of one patient was excluded due to failure to pass the quality control (Supplementary Figure 1). Apart from the 18 patients, other patients received therapy other than combined immunotherapy or in other hospitals and therefore were not included in this analysis. This study is part of the registered study ChiCTR1900023914.

\section{Sample Preparation, Targeted NGS and Data Processing 605-Gene NGS Sequencing}

For the FFPE samples, ten $5 \mu \mathrm{m}$ tumor slices were used for DNA extraction using the QIAamp DNA FFPE Kit (QIAGEN, Valencia, CA, USA) following the manufacturer's instructions. DNA from peripheral blood lymphocytes (PBLs) was extracted using the RelaxGene Blood DNA system (Tiangen Biotech Co., Ltd., Beijing, China) according to the manufacturer's instructions. DNA from fresh tissue samples was extracted using the EasyPure ${ }^{\circledR}$ Genomic DNA Kit (Beijing TransGen Biotech, Beijing, China). DNA was quantified with the Qubit 2.0 Fluorometer and the Qubit dsDNA HS assay kit (Thermo Fisher Scientific, Inc., Waltham, MA, USA) according to manufacturer's instructions. In brief, fragmented genomic DNA underwent end-repairing, A-tailing and ligation with indexed adapters sequentially, followed by size selection using Agencourt AMPure XP beads (Beckman Coulter Inc., Brea, CA, USA), and DNA fragments were used for library construction using the KAPA Library Preparation kit (Kapa Biosystems, Inc., Wilmington, MA, USA) according to the manufacturer's protocol. Hybridization-based target enrichment was carried out with HaploX pan-cancer 605-gene NGS panel (gene list in Supplementary Table 1) and Pre.LM.PCR Oligos (Kapa
Biosystems, Inc.) in $50 \mu \mathrm{L}$ reactions, and 7-8 PCR cycles were performed depending on the amount of DNA input. DNA sequencing was then performed on the Illumina Novaseq 6000 system according to the manufacturer's recommendations at an average depth of $5000 \times$ for tissue.

Sequencing data were de-multiplexed and aligned to the hg19 genome (GRch37) using Burrows-Wheeler Aligner (http://bio-bwa.sourceforge.net/) version 0.7.15.r1140 using default settings. Pileup files for properly paired reads with mapping quality $\geq 60$ were generated using Samtools (http:// www.htslib.org/). Somatic variants were determined using VarScan2 (http://varscan.sourceforge.net/). Allele frequencies were calculated for all Q30 bases. Using a custom Python script, previously identified tumor DNA mutations were intersected with a Samtools mpileup file generated for each sample, and the number and frequency were then calculated for each mutation. A mutation was identified if $\geq 5$ mutant reads were identified and $\geq 1$ mutant read was identified on each strand. Matched genomic DNA from white blood cells was used as control. Copy number changes were analyzed using the DECoN software (https://github. com/RahmanTeam/DECoN).

\section{RNA-Seq}

RNA from fresh tissue samples was extracted using EasyPure $^{\circledR}$ RNA Kit (Beijing TransGen Biotech, Beijing, China). RNA was quantified with the Qubit 2.0 Fluorometer and the Qubit RNA HS assay kit (Thermo Fisher Scientific, Inc., Waltham, MA, USA) according to manufacturer's instructions. After ribosomal RNA (rRNA) was digested by TruSeq Stranded Total RNA, the rRNAremoved RNA was cut into small fragments for cDNA synthesis. Libraries were generated using the KAPA Library Preparation kit (Kapa Biosystems, Inc., Wilmington, MA, USA) according to the manufacturer's protocol. Sequencing was conducted with the Illumina Novaseq 6000 platform. Data processing for differential RNA expression analysis between tumor tissues and normal tissues was performed using the LIMMA R package. Expression with $|\operatorname{LogFC}|>1$ (FC stands for fold change) and adjusted $\mathrm{P}$ value (false discovery rate control, FDR) $<$ 0.05 were considered to be significant differential expression. The integrated dysregulated gene lists were saved for subsequent analysis.

\section{TCR-Seq}

DNA from peripheral blood lymphocytes (PBLs) was extracted using the RelaxGene Blood DNA system 
Table I Clinicopathological and Therapeutic Information for All Patients in This Study

\begin{tabular}{|c|c|c|c|c|c|c|}
\hline Patient ID & Sex & Age & BCLC Staging & Differentiation & Therapy & Response \\
\hline 001 & Male & 47 & C & Moderate & Sorafenib+Sintilimab & PR \\
\hline 002 & Female & 52 & C & Moderate & & $\mathrm{N} / \mathrm{A}$ \\
\hline 003 & Male & 49 & C & Moderate & Lenvatinib+Sintilimab & SD \\
\hline 004 & Male & 50 & C & Moderate & Lenvatinib+Sintilimab & PD \\
\hline 005 & Male & 58 & C & Moderate & Lenvatinib+Sintilimab & SD \\
\hline 006 & Male & 60 & C & Moderate & & $\mathrm{N} / \mathrm{A}$ \\
\hline 007 & Male & 46 & C & Moderate & Sorafenib+Sintilimab & PD \\
\hline 008 & Male & 48 & C & Low & Lenvatinib+Sintilimab & PD \\
\hline 009 & Male & 63 & B & Moderate & Sorafenib+Sintilimab & PD \\
\hline 010 & Male & 71 & C & Moderate & Sorafenib+Sintilimab & SD \\
\hline 011 & Male & 50 & C & Moderate & & $\mathrm{N} / \mathrm{A}$ \\
\hline 012 & Male & 46 & C & Moderate & Lenvatinib+Pembrolizumab & PR \\
\hline 013 & Female & 68 & C & Low & Lenvatinib+Tislelizumab & PD \\
\hline 014 & Male & 68 & C & High & & $\mathrm{N} / \mathrm{A}$ \\
\hline 015 & Male & 33 & C & Moderate & Sorafenib+Sintilimab & PD \\
\hline 016 & Male & 68 & C & Moderate & & $\mathrm{N} / \mathrm{A}$ \\
\hline 017 & Male & 69 & C & Moderate & Lenvatinib+Sintilimab & PD \\
\hline 018 & Male & 54 & C & Moderate & Lenvatinib+Sintilimab & PR \\
\hline 019 & Male & 63 & B & Moderate & & N/A \\
\hline 020 & Female & 61 & C & Moderate & Sorafenib+Sintilimab & PR \\
\hline 021 & Male & 53 & C & High & Lenvatinib+Sintilimab & SD \\
\hline 022 & Male & 66 & C & Moderate & Lenvatinib+Sintilimab & SD \\
\hline 023 & Female & 36 & C & Moderate & & $\mathrm{N} / \mathrm{A}$ \\
\hline 024 & Male & 55 & C & Moderate & Lenvatinib+Sintilimab & SD \\
\hline 025 & Female & 56 & C & Moderate & Lenvatinib+Sintilimab & PR \\
\hline
\end{tabular}

(Tiangen Biotech Co., Ltd., Beijing, China) according to the manufacturer's instructions. Samples were analyzed by high-throughput sequencing of the TCR $\beta$ CDR3 region using the ImmunoSEQ immune profiling system (Adaptive Biotechnologies, Seattle, WA) at deep level. Sequencing was performed with Illumina Novaseq 6000 platform. In order to retain high-quality data for further analysis, FASTP software was used to filter the sequence. Samples in compliance with quality control (clonal reads $\geq 100 \mathrm{~K}$ ) were allowed for subsequent analysis. FLASH software was used to combine high-quality fragment data, and MiXCR software was then used to compare the fragment sequence with TCRV $\beta / D \beta / J \beta$ gene sequences in immune cell receptor library, and analyze the frequency of CDR3 clone subtype $\mathrm{V} \beta / \mathrm{D} \beta / \mathrm{J} \beta$ in each sample.

\section{Statistics, Data Analysis, Calculation of TMB and Molecular Subtyping}

Statistical analysis was performed and figures were plotted with GraphPad PRISM 5.0 software (GraphPad Software,
Inc, La Jolla, CA 92037, USA). Student's $t$-test was performed when two groups were compared, and ANOVA and post-hoc tests (Bonferroni test) were performed when three or more groups were compared. Chi-square test and Fisher test were performed when rate or percentage was compared for significance. Mutation spectrum and clustering analysis were conducted and figures were made with relevant packages of the $\mathrm{R}$ software (https://www.r-pro ject.org/).

\section{Results}

\section{Genomic Alterations of HCC and}

\section{Response Stratification Markers for the} First-Line TKI \pm Immunotherapy

In order to study the potential stratification of genetic alterations on HCC immunotherapy, we first studied the genomic variation of HCC. Figure 1A shows the mutation spectrum of HCC by the 605-gene panel. It was found that TP53, TERT, CSMD3, CTNNB1 and ARID1A genes were among the highly mutated genes in HCC. In addition, copy 


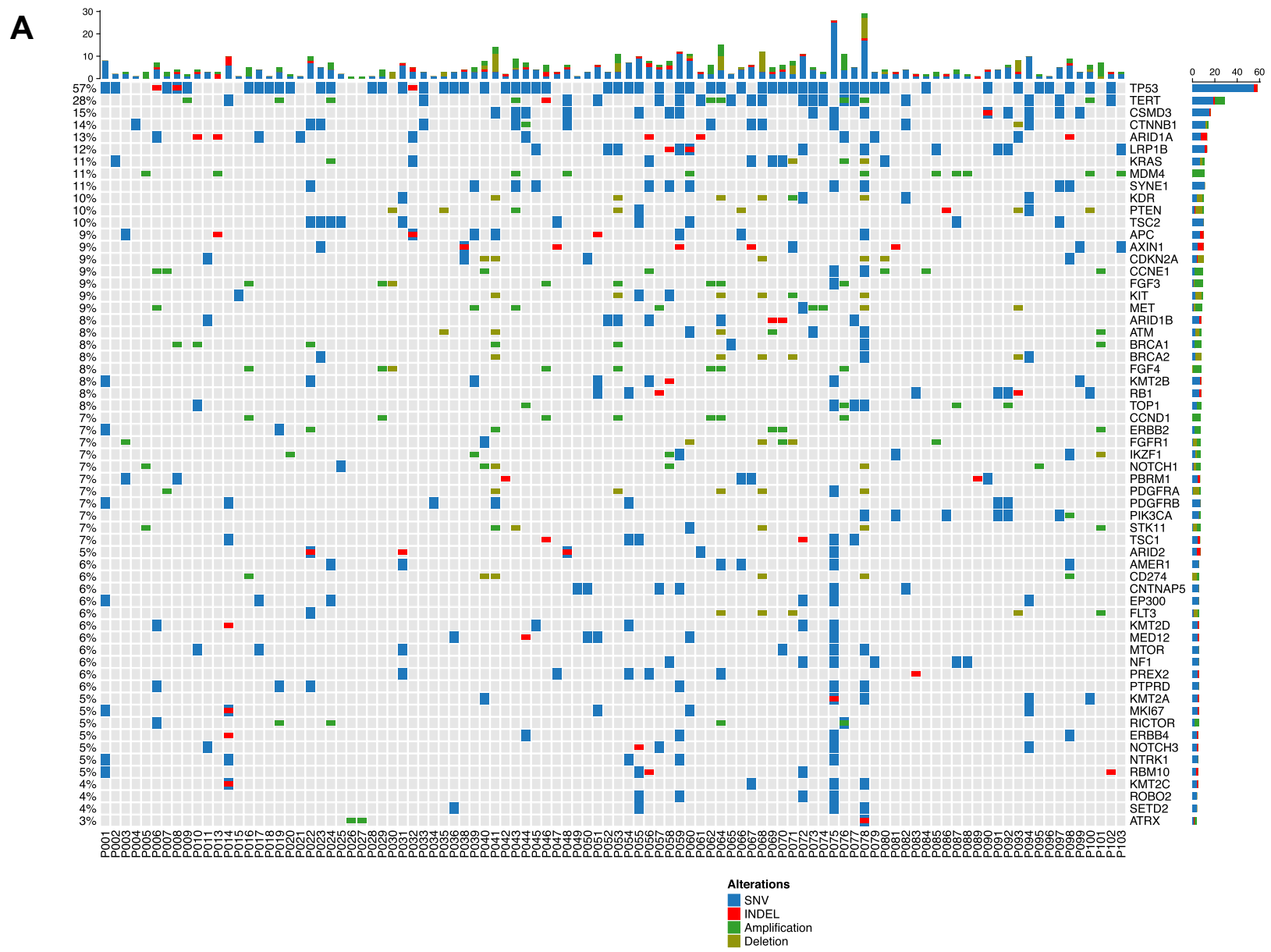

B
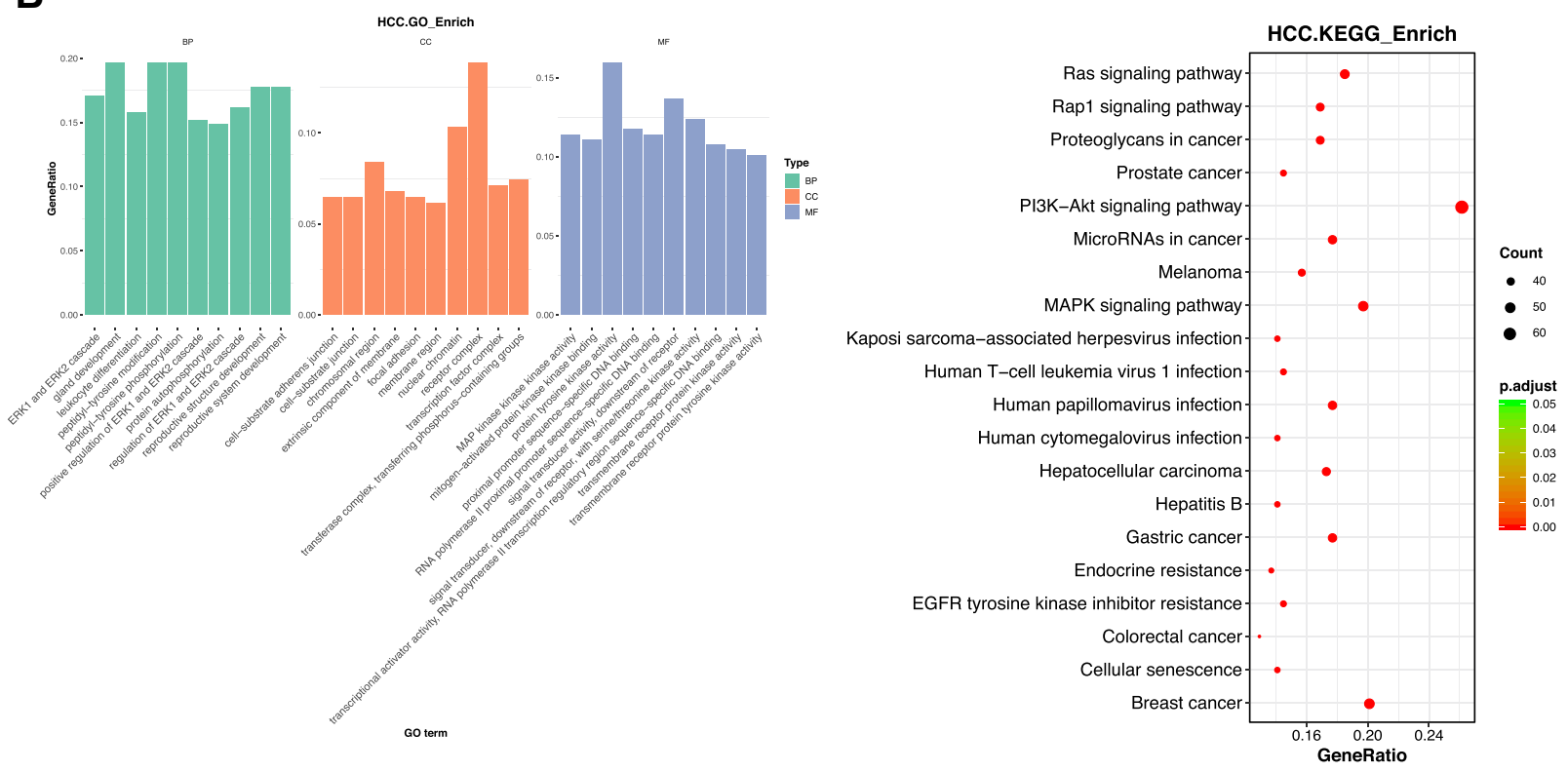

Figure I The genomic alterations of HCC. Panel (A) the alteration spectrum of $103 \mathrm{HCC}$ patients, including SNV, INDEL and CNV alterations. Panel (B) the results of GO (left) and KEGG (right) clustering analyses based on genomic alterations. In the left panel (GO clustering), green bars, Orange bars and blue bars represent enriched biological processes, cellular compartments and molecular functions, respectively, which are labeled below the figure. The Y-axis represents the ratio of genes that fall into the labeled items. In the right panel (KEGG clustering), the enriched pathway of functions are labeled on the left (Y-axis), and the ratio of genes fall into the labeled items is labeled below the figure. The size of dots represents the number of genes and the color represents the adjusted $p$ values. Bigger dots stand for higher count and red stands for lower $\mathrm{p}$ values. The major abnormalities included ERKI/2 pathway, cell membrane function, specific DNA binding, PI3K-AKT pathway, MAPK pathway, RAS pathway, RAPI pathway and HPV infection. 
number variations (CNVs) were also identified in genes, including TERT, MDM4, FGF3/4/19 and MET. The full list of variations detected by the 605-gene panel is summarized in Supplementary Table 2. The results for GO and KEGG clustering analyses are shown in Figure 1B, illustrating the aberrancies in major functions and pathways. It can be observed that the major abnormalities included ERK1/2 pathway, cell membrane function, specific DNA binding, PI3K-AKT pathway, MAPK pathway, RAS pathway, RAP1 pathway and HPV infection. We analyzed the correlation between gene alterations and the response of first-line immunotherapy in patients with HCC (Figure 2, Table 1). It was found that high-frequency mutations such as TP53 and TERT had no stratification effect on response, while MDM4 gene amplification had obvious stratification on response, in which a significantly higher percentage of MDM4 amplification was found in patients with cancer progression (PD) $(\mathrm{P}=0.018)$, while there no MDM4 amplification was found in partial response (PR) and stable disease (SD) patients. Further analysis revealed that FGF3/ FGF4/FGF19 genes were simultaneously amplified in two PD patients $(P=0.14)$, suggesting potential similar stratification to MDM4 amplification. We further conducted analysis on tumor mutational burden (TMB), and found no significant difference in TMB between PR/SD and PD patients (Figure 2).

\section{Transcriptional Alterations of HCC and Response Stratification Markers for the First-Line Immunotherapy}

To further investigate the relationship between HCC variation and the response of combined first-line immunotherapy, we collected the cancer and paracancerous normal tissue from HCC patients, and examined the mRNA and
lncRNA level alterations in HCC by RNAseq. Our study revealed 501 significantly differentially expressed mRNAs and 882 significantly differentially expressed lncRNAs (Figure 3). Further analysis showed that significant differences of mRNA were mainly present in synaptic function, cell membrane functions, systemic lupus erythematosus (SLE), cell division and chromosome and RNA polymerase (Figure 3A), in which mRNA significantly upregulated in chromosome function, SLE, endoplasmic reticulum function, alcoholism, cell cycle and cell division (Supplementary Figure 2A), and significantly downregulated in the cell membrane function and cell adhesion (Supplementary Figure 2B). Significant differences of lncRNA were mainly present in nervous system development, ion channel function, cAMP pathway, MARK pathway, RAS pathway, Wnt pathway, calcium signaling, neuroactive ligand-receptor interaction and cell adhesion (Figure 3B), in which lncRNA significantly up-regulated in nervous system development, an ion channel function, cAMP pathways and neuroactive ligand-receptor interaction (Supplementary Figure 2C), and significantly downregulated in cell adhesion, cAMP pathway, neuroactive ligand-receptor action and membrane proteins (Supplementary Figure 2D).

We further compared the levels of these differentially expressed mRNAs/lncRNAs between PR/SD and PD groups, and the results showed that the mRNA levels of 6 genes and the corresponding levels of 2 lncRNAregulated genes were significantly different between PR/ $\mathrm{SD}$ and PD groups. These included the mRNA levels of DGKI, TNFSF14, CHST4, ACTIN1, PFKP and SLC51B (Figure 4A) and the lncRNA levels of LCK and ERN1 (Figure 4B). These results suggest that the mRNA or lncRNA levels of these genes had potential roles in stratifying the response of the first-line combined
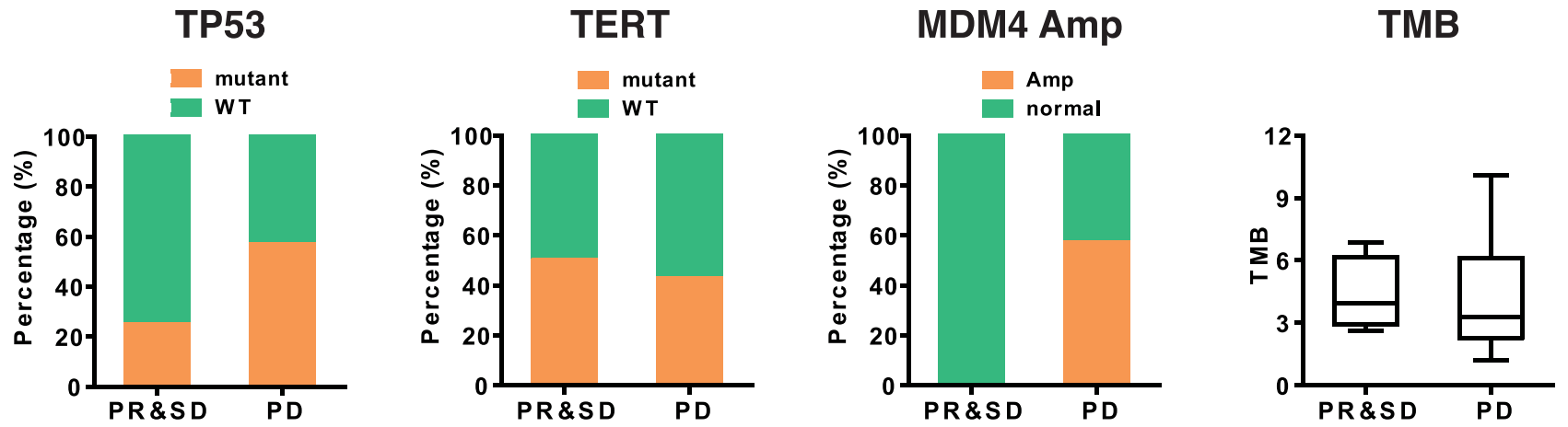

Figure 2 The percentage of major genetic alterations and TMB in PR/SD and PD groups. The percentage of patients with alterations or WT is shown for TP53, TERT and MDM4. The TMB for the two groups are also shown as indicated. 

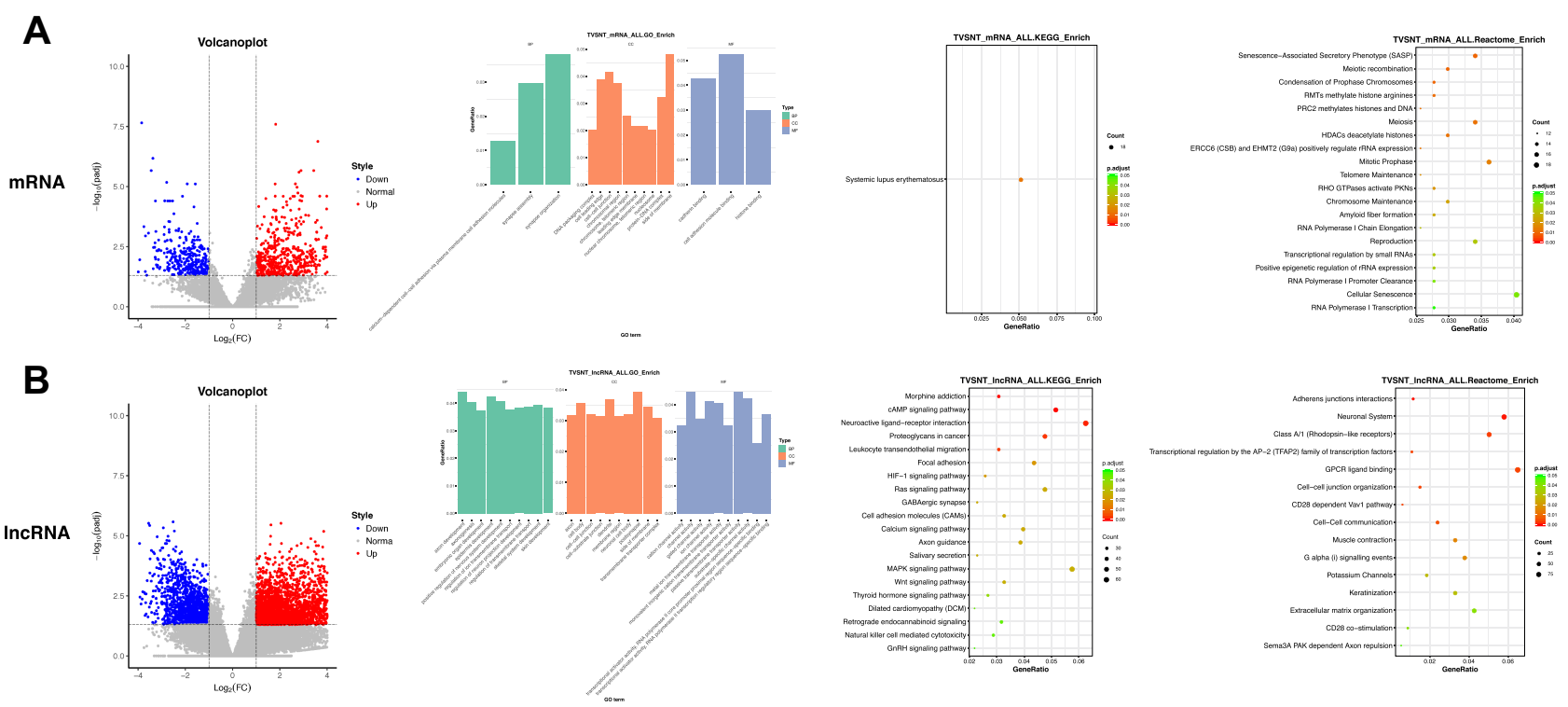

Figure 3 The alterations of mRNA and IncRNA in HCC compared with normal tissue. Significant up-regulations (red dots) and down-regulations (blue dots) in mRNA (panel $(\mathbf{A})$ ) and IncRNA (panel (B)) are presented as volcano plots. Read dots represent significantly upregulated genes and blue dots represent significantly downregulated genes and grey dots represent insignificant changes (normal). P $<0.05$ and Log2(fold change, FC) $\leq-1$ or $\geq I$ were selected as the thresholds for significant change. Clustering analyses for these significant alterations (both up- and down-regulations) are performed and presented as GO, KEGG and Reactome clustering, as indicated in each panel. The interpretation of GO, KEGG and Reactome clustering figures is identical to that describe in legends of Figure I. Significant differences of mRNA were mainly present in synaptic function, cell membrane functions, systemic lupus erythematosus (SLE), cell division and chromosome and RNA polymerase (Figure 3A). Significant differences of IncRNA were mainly present in nervous system development, ion channel function, cAMP pathway, MARK pathway, RAS pathway, Wnt pathway, calcium signaling, neuroactive ligand-receptor interaction and cell adhesion, (Figure 3B).

immunotherapy. We further compared our findings in mRNA with those recorded in the TCGA database, including data from $371 \mathrm{HCC}$ tissue samples and 50 adjacent normal tissue samples. Database analysis of HCC and adjacent normal tissue revealed 8515 significant differentially expressed genes between $\mathrm{HCC}$ and normal tissues, in which DGKI $(\mathrm{P}=1.99 \mathrm{E}-30)$, CHST4 $(\mathrm{P}=1.08 \mathrm{E}-17)$, PKFP $(\mathrm{P}=1.19 \mathrm{E}-5)$ and SLC51B $(\mathrm{P}=$ $1.43 \mathrm{E}-15)$ were the overlapping genes compared with our findings. This observation supported the validity of our findings.

Immune factors play important roles in immunotherapy, especially in the recognition of $\mathrm{T}$ cell chemotaxis and tumor killing. We therefore studied the correlation between a panel of immune factors (including BTLA, CCL5, CD34, CD96, CXCL9, CXCL10, ICAM1, LAG3TIGIT, TNF, VCAM1, VEGFA, VEGFB) and the therapeutic response, and found no significant correlation between response and the expression of these immune factors. We further investigated the correlation between the mRNA levels of these immune factors and the mRNA/lncRNA levels of the identified genes with potential response stratification effect. The results showed significant correlations between TIGIT, CD34 and CHST4 mRNA levels, between ICAM1 and SLC51B
mRNA levels, and between CCL5, CXCL9, CXCL10 and ERN1 mRNA levels (Figure 5). These results suggest that although these immune factors were not able to stratify the response directly, their expression correlated with the mRNA/lncRNA levels of genes that stratify the response, and therefore could correlate with the response indirectly.

\section{Immune Repertoire Alterations of HCC and Potential Indications of Response Stratification}

We further studied the TCR repertoire diversity of HCC by defining the three commonly used indicators. We found that Shannon-Wiener Index and Evenness fluctuated significantly among different patients, while Simpson index was more uniform (Figure 6A). The correlation study of the three indicators revealed that any two of the three indicators were significantly correlated, indicating a good consistency for diversity assessment using different indicators (Figure 6B). We then compared the levels of each of the three indicators between $\mathrm{PR} / \mathrm{SD}$ and $\mathrm{PD}$ groups. Figure 7A showed that Shannon-Wiener index, Simpson index and evenness had no significant difference between $\mathrm{PR} / \mathrm{SD}$ and PD groups, suggesting no stratification on 
A
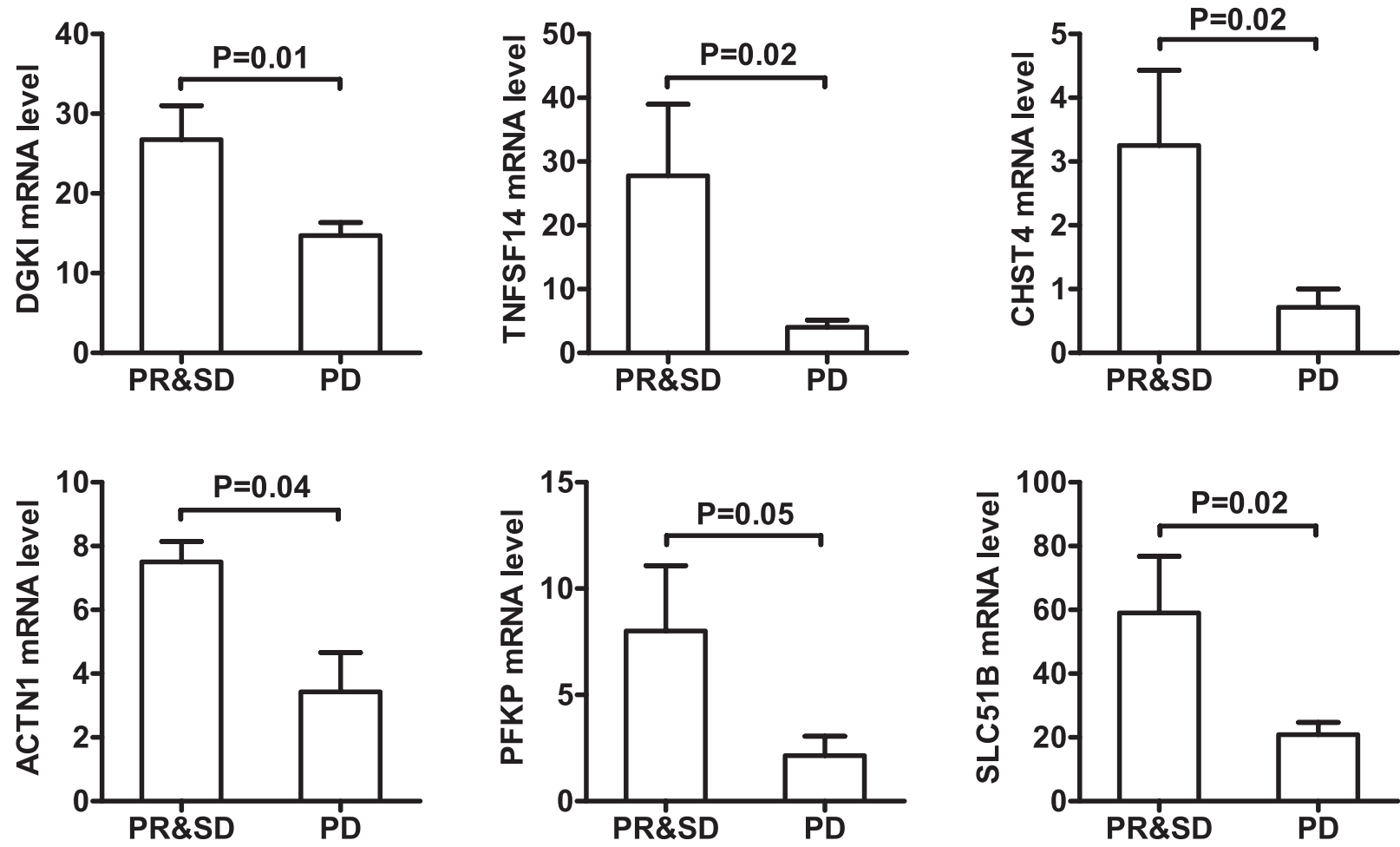

B
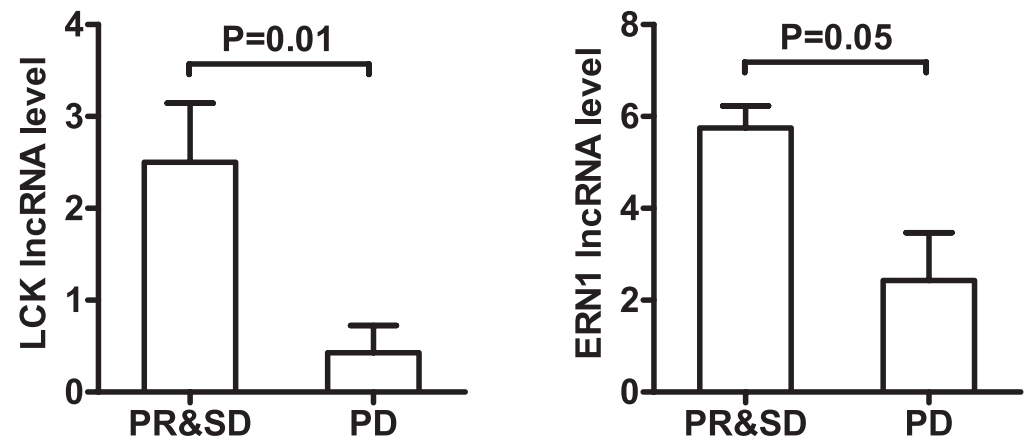

Figure 4 Significant differences in mRNA and IncRNA levels of individual genes were observed between PR/SD and PD groups. The differences in mRNA levels between PR/ SD and PD groups for six genes are presented in panel (A) and the differences in IncRNA levels between PR/SD and PD groups for two genes are presented in panel (B). $P$ values are labeled for each individual gene. Data are presented as mean with standard deviation (SD).

response. Further analysis showed correlation between the three indicators and the mean RNA level of all genes obtained from the tumor tissue sample of certain patient (Figure 7B), in which Shannon-Wiener index and evenness showed a weak negative correlation with the mean RNA level (Figure 7B), indicating that TCR CD3 diversity in tumor tissues possible negatively correlated with RNA level. We further performed the stratification analysis on J, $\mathrm{V}$ and $\mathrm{V}-\mathrm{J}$ subtypes, in which 12 patients agree to provide individual data on J, V and V-J subtypes (Supplementary Figure 3). Individual data of J subtypes (Supplementary
Figure 3A), V subtypes (Supplementary Figure 3B) and V-J subtypes (Supplementary Figure 3C) were obtained from individual patients, as shown in Supplementary Figure 3. All patients were divided into two groups by response (PR/SD and PD). Statistics did not show any significant difference in subtype distribution between PR/ SD and PD group, possibly due to the limited number of patients in this analysis. Variation of subtype distribution among patients can be observed, and the pattern of $\mathrm{J}$, $\mathrm{V}$ and V-J subtype distribution within the same group appeared to be similar across most individuals. 

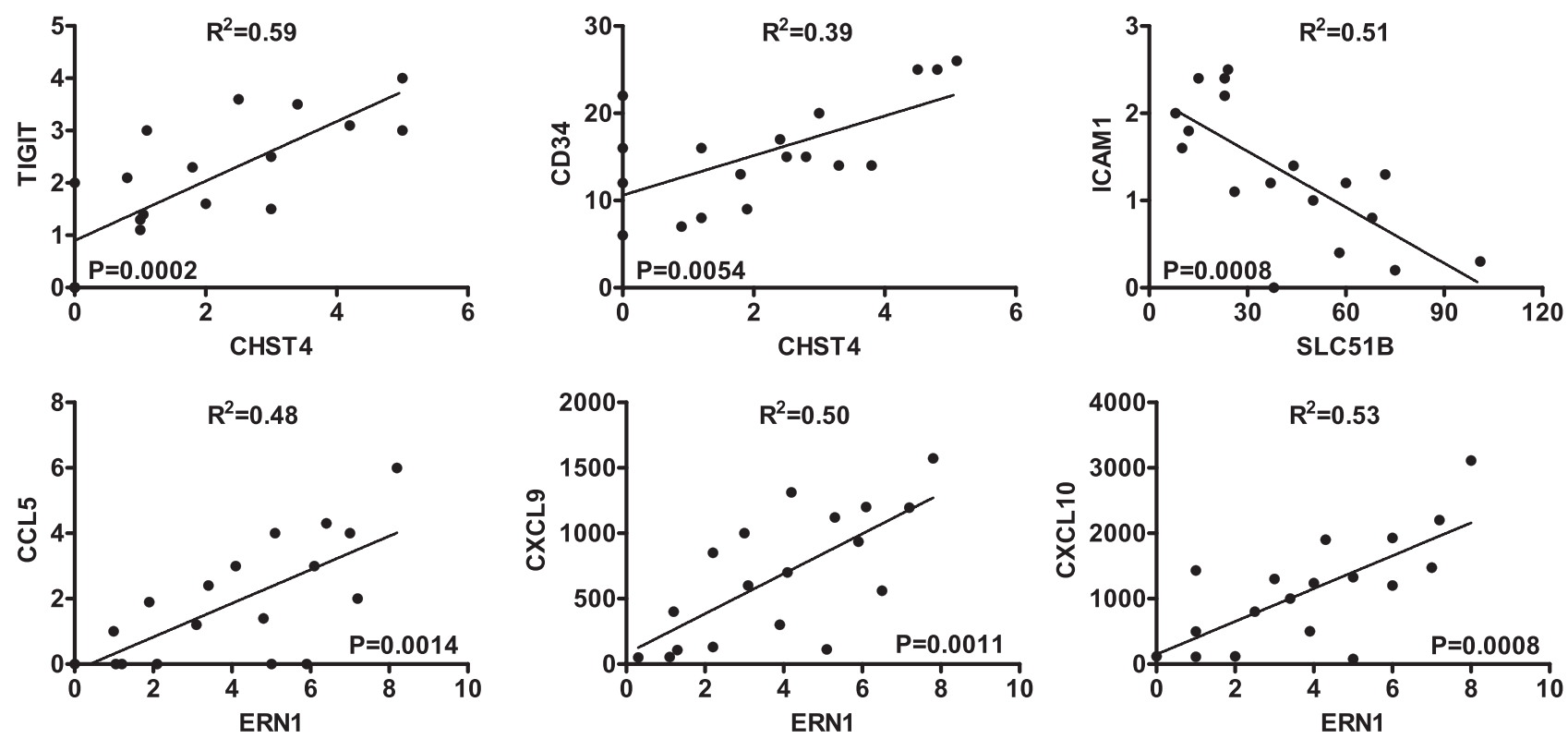

Figure 5 Correlation analyses between individual mRNA and immune factors. Significant linear correlation was found between pairs of mRNA and immune factors. Coefficient of determination $\left(R^{2}\right)$ and $P$ values are labeled.

\section{Discussion}

The first-line combined immunotherapy for HCC has been recommended by the 2020 NCCN guidelines and newly reported in 2020 ASCO annual meeting. Currently, it mainly involves two strategies, including atezolizumab +bevacizumab $(\mathrm{T}+\mathrm{A})^{10}$ and pembrolizumab+lenvatinib $(\mathrm{K}+\mathrm{L}) .{ }^{13}$ The FDA approval of $\mathrm{T}+\mathrm{A}$ therapy was based on a multicenter, open-label, randomized, phase III study (IMbrave150). The median PFS was significantly longer in the combined group than in the sorafenib group (6.8 months vs 4.3 months; HR $=0.59$ [95\% CI 0.47-0.76]; $\mathrm{P}<0.0001)$. Median OS was not achieved in the combined group, and was 13.2 months in the Sorafenib group (HR 0.58 [95\% CI 0.42-0.79]; $\mathrm{P}=0.0006$ ). According to RECIST 1.1, the ORR in the combination group was more than twice that of the sorafenib group $(27 \%$ vs $12 \%$; $(\mathrm{p}<0.0001))$. The median duration of treatment for combined therapy was 7.4 months versus 3 months for sorafenib. ${ }^{10}$ This is the first treatment in more than a decade to show improved survival in untreated patients with advanced HCC.

Pembrolizumab+lenvatinib $(\mathrm{K}+\mathrm{L})$, another combined therapy for advanced $\mathrm{HCC}$ also exhibited promising prospect. The KEYNOTE-524 study (NCT03006926) was a non-blind, multicenter, IB phase trial evaluating tolerability and safety. ${ }^{13}$ One hundred and four patients with BCLC stage B or C HCC (Child-Pugh class A, ECOG 0 or 1) without prior systemic therapy were enrolled. No dose-limiting toxicity (DLTs) was observed in 45 patients, and the enrollment was expanded to 104 patients (DLT, $\mathrm{n}=6$; Upgrade, $\mathrm{n}=98$ ). At a mean follow-up of 10.6 months, 37 patients remained on treatment (lenvatinib, $\mathrm{n}=$ 3; Combination, $\mathrm{n}=34$ ). According to RECIST v1.1, ORR was 36\% (95\% CI: 26.6-46.2), including $1 \mathrm{CR}(1 \%)$ and 35 PR (35\%). These results were better than those of the lenvatinib group (ORR: 24.1\%, 6 CR (1\%) and 109 PR (23\%)). The median PFS of 19 patients was 8.6 months (95\% confidence interval, 7.1-9.7) and the median DOR was 12.6 months $(95 \%$ confidence interval, 6.9-not estimable). ${ }^{14} \mathrm{~A}$ multicenter, double-blind, phase III trial, LEAP-002 (NCT03713593) is currently investigating the efficacy of this combination in first-line patients with advanced HCC (Child-Pugh class A). ${ }^{15}$

Based on the approval by FDA and the above clinical trial data, sorafenib+sintilimab $(\mathrm{S}+\mathrm{S})$ and lenvatinib + sintilimab $(\mathrm{L}+\mathrm{S})$ were used in this study. Sorafenib targets CRAF, BRAF, BRAF V600E, C-KIT, FLT-3, VEGFR-2, VEGFR-3, PDGFR- $\beta$ and lenvatinib targets VEGFR-1, VEGFR-2, VEGFR-3, FGFR1, PDGFR, CKIT, RET, etc. Since sorafenib and lenvatinib are both multi-target inhibitors with common targets, we combined the two strategies as one group for analysis in this study. Our study found that high-frequency driver gene mutations in HCC, including TP53 and TERT, did not stratify response, and similarly, TMB did not stratify response. However, it has 


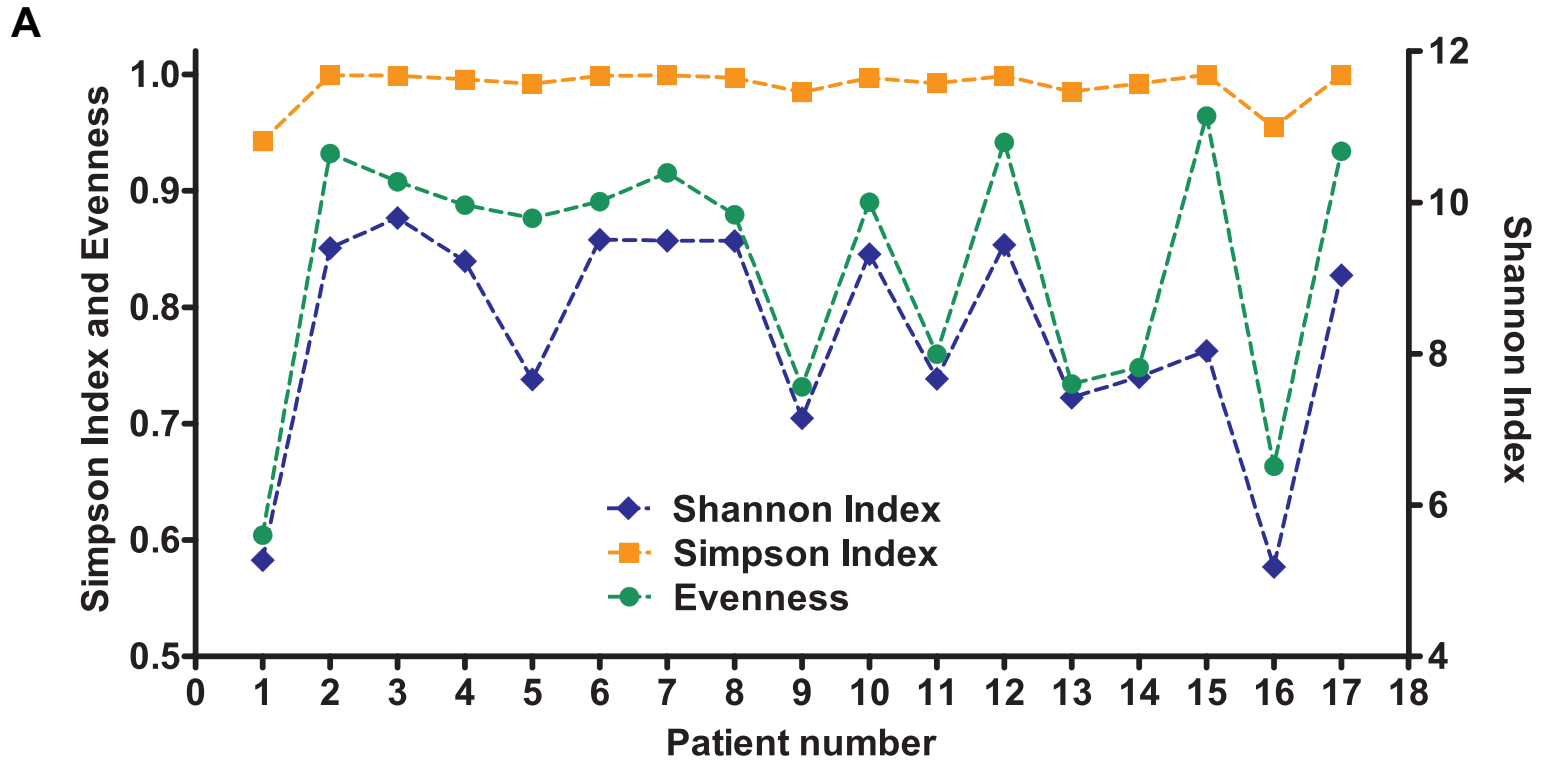

B
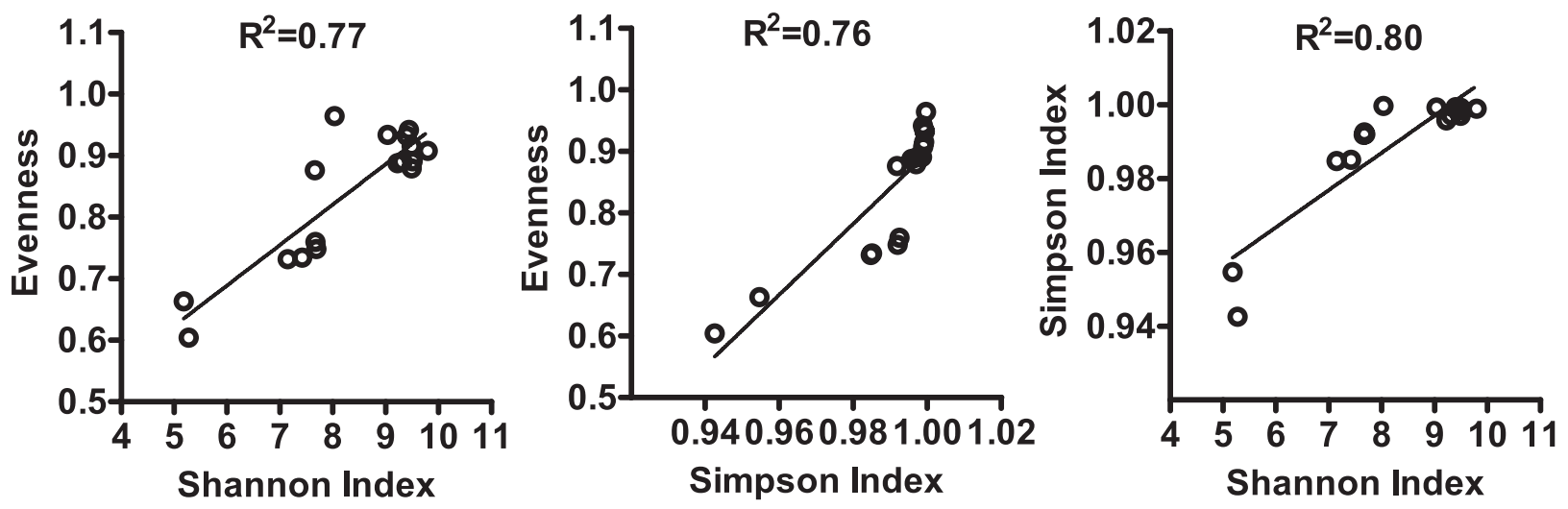

Figure 6 Assessment of TCR CDR3 diversity and the correlation of indexes. Panel (A): Shannon-Wiener Index, Simpson index and evenness for I7 HCC patients. Individual values are connected to compare the trend of different indexes. Panel (B): strong correlation was revealed between any two of the three indexes.

been reported in previous literatures that high-frequency mutations such as TP53 and CTNNB1 were closely associated with TMB-high, ${ }^{16}$ and patients carried TP53 neoantigen had a longer overall survival than others. ${ }^{17}$ Mutations in TERT, CTNNB1, BRD4 or MLL, and co-mutations in TP53 and TERT or BRD4 were associated with significantly worse survival. ${ }^{18}$ Furthermore, several studies showed that patients with high TMB exhibited worse OS than those with low TMB. ${ }^{18-22}$ However, all the above studies were from single-drug immunotherapy at first-line or multiple lines, and the stratification effect of mutation and TMB in the first-line combined immunotherapy on response and prognosis has not been reported. Since immunotherapy by single drugs is not as effective as combined therapy, our observations in this study may lead to the speculation that the combination of PD-1 blockade with multi-target inhibitors may diminish the stratification effect of individual mutations and TMB, as the improvement of overall response may obscure the effect of biomarkers.

Although mutations and TMB were unable to stratify the response HCC in first-line combined immunotherapy, MDM4 amplification was found to be an effective predictive marker for disease progression in patients. Similarly, FGF3/4/19 amplification may also be a predictive marker for $\mathrm{PD}$ in patients. MDM2/MDM4 amplification was reported to be associated with cancer hyperprogression disease (HPD) in other cancers but has not been reported in HCC immunotherapy. ${ }^{23-27}$ Similarly, FGF3/4/19 amplification was also reported to be predictive markers for 
A
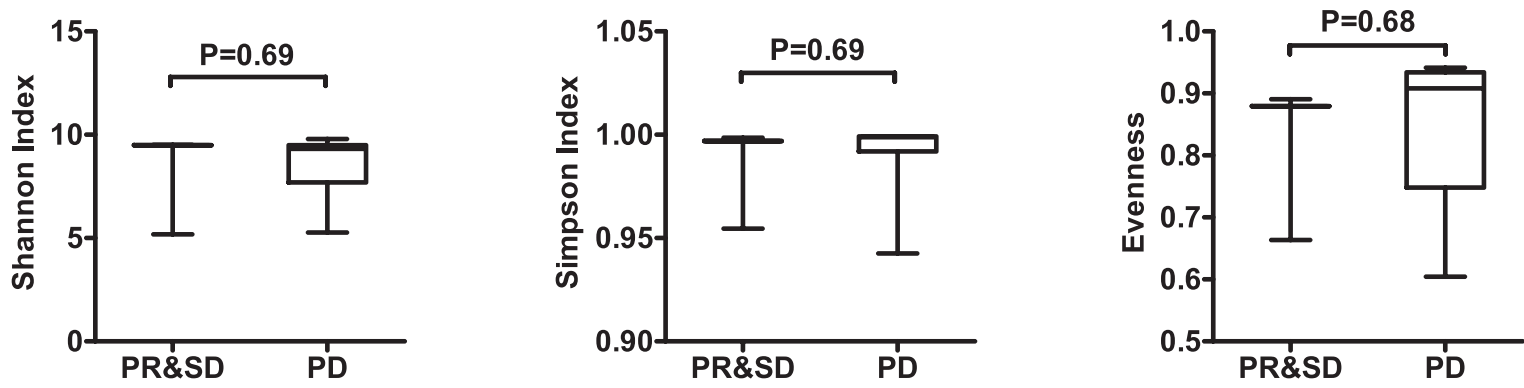

B
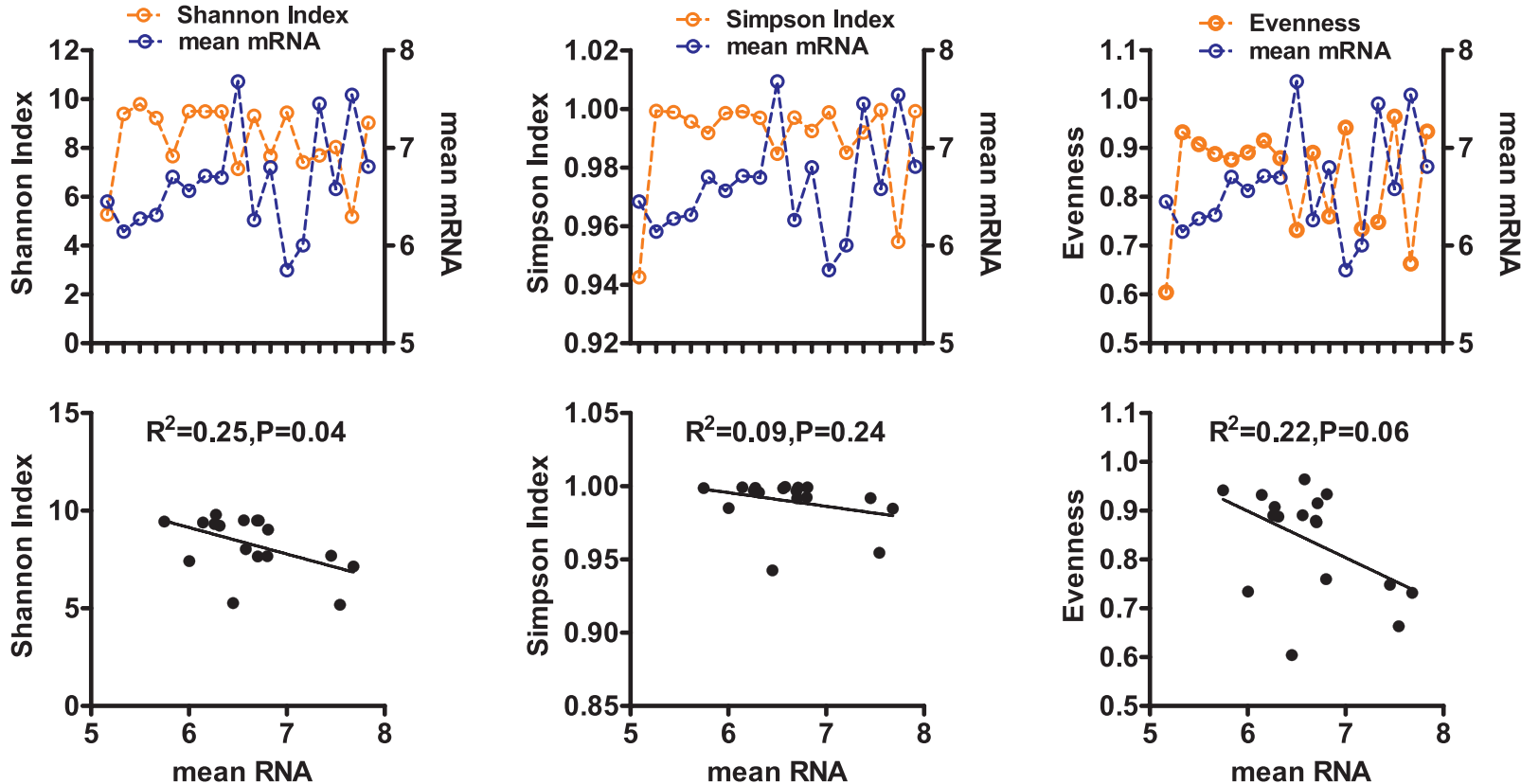

Figure 7 Stratification of TCR CDR3 diversity and its correlation with mean mRNA. Panel (A): Box-and-Whisker's plot for Shannon-Wiener Index, Simpson index and evenness in PR/SD and PD groups. Panel (B) upper row: individual values show the correlation between the three indexes and the mean RNA levels. Individual values are connected to compare the trend of different indexes in the figures of the upper row. Panel (B) lower row: linear correlation was found between Shannon index and mean RNA level, and between evenness and the mean RNA level.

HPD in HCC and other cancers at multiple lines of treatment. $^{28-33}$ Therefore, MDM4 and FGF3/4/19 amplification are very likely to be biomarkers for disease progression in first-line combined immunotherapy for $\mathrm{HCC}$, similar to previous studies with multiline immunotherapy or targeted therapy.

In this study, we identified 8 genes that had stratifying capability on first-line combined immunotherapy. Functional classification of these genes showed that they were mainly involved in liver metabolism regulation (DGKI, ACTIN1, PFKP, SLC51B) and tumor immune regulation (TNFSF14, CHST4, LCK, ERN1). It appeared that the abnormal levels of mRNA or lncRNA of these genes reflected the abnormal changes in metabolism and immune regulation in $\mathrm{HCC}$, and were significantly associated with response. Interestingly, the mRNA or lncRNA levels of these genes were significantly higher in the PR/ SD group than in the PD group, suggesting that high mRNA/lncRNA levels were good predictors of response, while no genes at low levels were found to correlate with better efficacy. Therefore, these 8 genes could constitute a panel that could be used as predictors of response in first-line combined immunotherapy. Due to the limited number of patients, these 8 genes were not verified in this study, and subsequent studies should establish a model and verify it. We further analyzed the expression of a series of immune factors known to play roles in tumor cell recognition and specific killing. Our results showed that none of these immune factors showed a stratification effect, we therefore analyzed the correlation between these immune factors and the 8 genes correlated with response, and found that 6 immune factors exhibited moderate 
correlations. These observations suggest that although immune factors themselves could not significantly stratify response, they were significantly correlated with genes stratifying response (CHST4, SLC51B, and ERN1), suggesting that they indirectly correlated with response. These immune factors not only involve those related to immune cell function, such as TIGIT, cell surface antigen recognition molecules, such as CD34 and ICAM1, but also involve chemokines such as CCL5, CXCL9 and CXCL10. Therefore, the above observations were helpful to clarify the relationship between response stratification genes and immune factors and helped to explain the roles of immune factors in first-line combined immunotherapy.

Immune repertoire, especially the $\mathrm{CDR} 3$ region of TCR, has been studied in cancers including HCC. The immune repertoire reflects an aspect of the tumor microenvironment, representing the degree of clonal diversity in the T-cell receptor variable region CDR3. In cancers besides $\mathrm{HCC}, \mathrm{CDR} 3$ region diversity is considered as an indicator of immunotherapeutic efficacy. For example, in patients with lung cancer underwent surgery, high $\mathrm{T}$ cell clonality was considered to be associated with poor postoperative survival, ${ }^{34}$ and may also be associated with higher recurrence. ${ }^{35}$ Furthermore, dynamic detection of the TCR repertoire during anticancer treatment was useful for prognosis. The degree of overlap and trend of alteration in the diversity of TCR CDR3 region before and after treatment may affect the prognosis of patients. ${ }^{36}$ In another report on melanoma, patients treated with CTLA4 blockade experienced a significant increase in the number and complication of TCR variants, which was attributable to the drug treatment. ${ }^{37}$ Although the study found no association between baseline TCR diversity and response to CTLA-4 blockade, other studies showed association between high TCR repertoire diversity before therapy and clinical benefit. $^{38}$ Since the baseline TCR repertoire diversity of patients before treatment may vary greatly due to individual differences, it appears that the changes but not the absolute values of the TCR repertoire diversity during treatment may be an indicator of the response and prognosis of patients. In HCC immunotherapy, it is lack of similar study on the stratification of response by TCR Repertoire. In this study, we examined the peripheral blood TCR repertoire diversity and measured it with three commonly used indicators. We found that the three indicators had strong linear correlation, indicating good consistency among the indicators. Simpson index fluctuated less, while Shannon-Weiner index and Evenness fluctuated substantially among individuals, indicating some differences existed in the three indexes. We did not observe stratification effect of TCR repertoire diversity on patient outcomes, which could be due to a number of factors. First, we measured the TCR repertoire diversity of pretherapeutic blood samples from patients. As mentioned above, the absolute value of TCR repertoire diversity may be not necessarily related to response, while changes during therapy may be related to response. However, we were unable to obtain post-therapeutic tissue samples from patients in this study. Secondly, it is unclear whether the TCR repertoire diversity alone can accurately reflect the immune status of HCC patients, because it is affected by many factors, such as age. ${ }^{39}$ Thirdly, more evidence is still needed on whether blood TCR repertoire diversity can accurately reflect HCC TCR repertoire diversity. Furthermore, we found that the changes of ShannonWeiner index and evenness were weakly negatively correlated with the mean RNA range of patients, indicating that the individual TCR repertoire diversity of HCC patients may be correlated with the individual RNA level. We therefore speculate that the changes of TCR repertoire diversity during immunotherapy may be relatively independent but correlated with the changes of total RNA to a certain extent. More research is needed to clarify the exact correlation.

Recent studies supported the notion that specific T-cell populations are present and may be related to HCC response to immunotherapy, and therefore T-cell subtyping could be used in patient selection or response stratification. ${ }^{40-45}$ This mainly involved specific CD4+ and/or CD8+ T-cells that can infiltrate the HCC cancer tissue. The findings could also be true in PD-1 inhibitor combined with multiple target inhibitors such as regorafenib ${ }^{45}$ or lenvatinib (this study). It is possible that T-cell clonality (TCR repertoire diversity), combined with specific T-cell markers (such as CD4, CD8) may be used to stratify patient response and prognosis. On the other hand, activation or expansion of specific T-cell types may be used to enhance the response to immunotherapy in future.

There were some limitations with this study. First, the number of patients was limited with first-line combined immunotherapy. This limited the power of analysis in stratification by mutations, amplifications, mRNA, lncRNA and TCR sequencing, and bias may exist with small number of cases. More cases should be collected in future to validate the findings in this study. Secondly, posttherapeutic samples should be collected as much as possible to study the alterations of mutations, transcriptions and 
TCR diversity changes after therapy, which could provide extra information on molecular alterations during first-line combined immunotherapy and help to find new biomarkers of response stratification. Thirdly, follow-up data should be collected to identify potential biomarkers for prognosis prediction.

\section{Abbreviations}

ASCO, American Society of Clinical Oncology; BCLC, Barcelona clinic liver cancer; cAMP, cyclic adenosine monophosphate; CDR3, complementarity determining region 3; CTLA4, Cytotoxic T lymphocyte antigen-4; DLTs, doselimiting toxicity; DNA, deoxyribonucleic acid; ECOG, Eastern Cooperative Oncology Group; ERK, extracellular regulated protein kinase; FDA, food and drug administration; FFPE, formalin-fix paraffin-embedded; GO, gene ontology; $\mathrm{HBV}$, hepatitis B virus; HCC, hepatocellular carcinoma; $\mathrm{HCV}$, hepatitis C virus; HPD, hyperprogression disease; $\mathrm{HR}$, hazard ratio; KEGG, Kyoto encyclopedia of genes and genomes; lncRNA, long non-coding ribonucleic acid; MAPK, mitogen-activated protein kinase; mRNA, messenger ribonucleic acid; NCCN, national comprehensive cancer network; NGS, next-generation sequencing; ORR, objective response rate; PBLs, peripheral blood lymphocytes; PD, progression disease; PD-L1, programmed death ligand 1; PFS, progression free survival; PI3K-AKT, phosphatidylinositol 3'-kinase-protein kinase B; PR, partial response; RECIST, response evaluation criteria in solid tumors; rRNA, ribosomal ribonucleic acid; SD, stable disease; SLE, systemic lupus erythematosus; TCR, T cell receptor; TMB, tumor mutational burden.

\section{Data Sharing Statement}

The datasets generated and/or analyzed during the current study are available from the corresponding author upon reasonable request.

\section{Ethics Approval and Consent to Participate}

This study was approved by the ethics committee of the Fifth Medical Center of the Chinese PLA General Hospital. Written informed consent was obtained from all patients.

\section{Consent for Publication}

Consent for publication was obtained from patients involved in this study.

\section{Acknowledgments}

We thank all patients and their relatives for supporting the study and thank all technicians in this study for processing the samples and technical support.

\section{Author Contributions}

Zhen Zeng, Yinying Lu and Lele Song designed the study. All authors contributed to the execution, acquisition of data, analysis and interpretation of data. All authors contributed to data analysis, drafting or revising the article, gave final approval of the version to be published, agreed to the submitted journal, and agree to be accountable for all aspects of the work. All authors reviewed and agreed on all versions of the article before submission, during revision, the final version accepted for publication, and any significant changes introduced at the proofing stage. All authors agree to take responsibility and be accountable for the contents of the article. Lele Song submitted the manuscript.

\section{Funding}

This study was supported by the National Science and Technology Major Project "Prevention and Control of Major Infectious Diseases including AIDS and Viral Hepatitis' Special Project "Development and Transformation of Hepatitis B-Related Hepatic Cancer Markers for Accurate Diagnosis and Treatment" (2018ZX10302205) subproject "Translational Study of Tissue Microenvironment on Metastasis, Recurrence and Intervention of Liver Cancer" (2018ZX10302205-001). This study was also supported by the Youth Project of National Natural Science Foundation of China (81702389). This study was also supported by the Special Funds for Strategic Emerging Industry Development of Shenzhen (grant number 20170922151538732), the Science and Technology Project of Shenzhen (grant number JSGG20180703164202084). All funders did not participate in the study design, study implementation, data collection, data analysis, data interpretation and manuscript writing of the study.

\section{Disclosure}

Xiaohui Wang, Jianfei Yao, Aifang Ge and Lele Song were employees of HaploX Biotechnology, who performed the NGS sequencing and data analysis in this study. The authors report no other conflicts of interest in this work. 


\section{References}

1. Villanueva A, Longo DL. Hepatocellular carcinoma. $N$ Engl J Med. 2019;380(15):1450-1462. doi:10.1056/NEJMra1713263

2. Sartorius K, Sartorius B, Aldous C, Govender PS, Madiba TE. Global and country underestimation of hepatocellular carcinoma (HCC) in 2012 and its implications. Cancer Epidemiol. 2015;39(3):284-290. doi:10.1016/j.canep.2015.04.006

3. Hartke J, Johnson M, Ghabril M. The diagnosis and treatment of hepatocellular carcinoma. Semin Diagn Pathol. 2017;34(2):153-159. doi:10.1053/j.semdp.2016.12.011

4. Pinter M, Jain RK, Duda DG. The current landscape of immune checkpoint blockade in hepatocellular carcinoma: a review. JAMA Oncol. 2021;7(1):113-123. doi:10.1001/jamaoncol.2020.3381

5. Sprinzl MF, Galle PR. Current progress in immunotherapy of hepatocellular carcinoma. J Hepatol. 2017;66(3):482-484. doi:10.1016/j. jhep.2016.12.009

6. Yau T, Hsu C, Kim TY, et al. Nivolumab in advanced hepatocellular carcinoma: sorafenib-experienced Asian cohort analysis [published correction appears in J Hepatol. 2019 Dec;71(6):1278]. J Hepatol. 2019;71(3):543-552. doi:10.1016/j.jhep.2019.05.014

7. Yau T, Park JW, Finn RS, et al. CheckMate 459: a randomized, multicenter phase III study of nivolumab (NIVO) vs sorafenib(SOR) as firstline (1L) treatment in patients (pts) with advanced hepatocellular carcinoma (aHCC). Ann Oncol. 2019;30(5):LBA38. doi:10.1093/annonc/mdz394.029

8. Finn RS, Ryoo BY, Merle P, et al. Pembrolizumab as second-line therapy in patients with advanced hepatocellular carcinoma in KEYNOTE-240: a randomized, double-blind, phase III trial. $J$ Clin Oncol. 2020;38(3):193-202. doi:10.1200/JCO.19.01307

9. Zhu AX, Finn RS, Edeline J, et al. Pembrolizumab in patients with advanced hepatocellular carcinoma previously treated with sorafenib (KEYNOTE-224): a non-randomised, open-label Phase 2 trial [published correction appears in Lancet Oncol. 2018 Sep;19(9):e440]. Lancet Oncol. 2018;19(7):940-952. doi:10.1016/S1470-2045(18) 30351-6

10. Finn RS, Qin S, Ikeda M, et al. Atezolizumab plus bevacizumab in unresectable hepatocellular carcinoma. $N$ Engl J Med. 2020;382 (20):1894-1905. doi:10.1056/NEJMoa1915745

11. Llovet J, Shepard KV, Finn RS, et al. A phase Ib trial of lenvatinib (LEN) plus pembrolizumab (PEMBRO) in unresectable hepatocellular carcinoma (uHCC): updated results[J]. Ann Oncol. 2019;30 (15):747P. doi:10.1093/annonc/mdz247.073

12. Qin S, Chen Z, Liu Y, et al. A phase II study of anti-PD-1 antibody camrelizumab plus FOLFOX4 or GEMOX systemic chemotherapy as first-line therapy for advanced hepatocellular carcinoma or biliary tract cancer. J Clin Oncol. 2019;37(15 suppl):4074. doi:10.1200/ JCO.2019.37.15_suppl.4074

13. Finn RS, Ikeda M, Zhu AX, et al. Phase Ib study of lenvatinib plus pembrolizumab in patients with unresectable hepatocellular carcinoma. J Clin Oncol. 2020;38(26):2960-2970. doi:10.1200/ JCO. 20.00808

14. Zhu A, Finn RS, Ikeda M, et al. A phase Ib study of lenvatinib (LEN) plus pembrolizumab (PEMBRO) in unresectable hepatocellular carcinoma (uHCC). J Clin Oncol. 2020;38(15_suppl):4519. doi:10.1200/ JCO.2020.38.15_suppl.4519

15. Llovet JM, Kudo M, Cheng AL, et al. Lenvatinib (len) plus pembrolizumab (pembro) for the first-line treatment of patients (pts) with advanced hepatocellular carcinoma (HCC): phase 3 LEAP-002 study. $J$ Clin Oncol. 2019;37:TPS4152-TPS4152. doi:10.1200/ JCO.2019.37.15_suppl.TPS4152

16. Li L, Rao X, Wen Z, et al. Implications of driver genes associated with a high tumor mutation burden identified using next-generation sequencing on immunotherapy in hepatocellular carcinoma. Oncol Lett. 2020;19(4):2739-2748. doi:10.3892/ol.2020.11372
17. Yang H, Sun L, Guan A, et al. Unique TP53 neoantigen and the immune microenvironment in long-term survivors of Hepatocellular carcinoma. Cancer Immunol Immunother. 2021;70(3):667-677. doi:10.1007/s00262-020-02711-8

18. Ou Q, Yu Y, Li A, et al. Association of survival and genomic mutation signature with immunotherapy in patients with hepatocellular carcinoma. Ann Transl Med. 2020;8(5):230. doi:10.21037/ atm.2020.01.32

19. Huo J, Wu L, Zang Y. A prognostic model of 15 immune-related gene pairs associated with tumor mutation burden for hepatocellular carcinoma. Front Mol Biosci. 2020;7:581354. doi:10.3389/ fmolb.2020.581354

20. Peng Y, Liu C, Li M, et al. Identification of a prognostic and therapeutic immune signature associated with hepatocellular carcinoma. Cancer Cell Int. 2021;21(1):98. doi:10.1186/s12935021-01792-4

21. Xie F, Bai Y, Yang X, et al. Comprehensive analysis of tumour mutation burden and the immune microenvironment in hepatocellular carcinoma. Int Immunopharmacol. 2020;89(Pt A):107135. doi:10.1016/j.intimp.2020.107135

22. Wong CN, Fessas P, Dominy K, et al. Qualification of tumour mutational burden by targeted next-generation sequencing as a biomarker in hepatocellular carcinoma. Liver Int. 2021;41(1):192-203. doi:10.1111/liv.14706

23. Forschner A, Hilke FJ, Bonzheim I, et al. MDM2, MDM4 and EGFR amplifications and hyperprogression in metastatic acral and mucosal melanoma. Cancers (Basel). 2020;12(3):540. doi:10.3390/ cancers 12030540

24. Adashek JJ, Subbiah IM, Matos I, et al. Hyperprogression and immunotherapy: fact, fiction, or alternative fact? Trends Cancer. 2020;6(3):181-191. doi:10.1016/j.trecan.2020.01.005

25. Kato S, Goodman A, Walavalkar V, Barkauskas DA, Sharabi A, Kurzrock R. Hyperprogressors after immunotherapy: analysis of genomic alterations associated with accelerated growth rate. Clin Cancer Res. 2017;23(15):4242-4250. doi:10.1158/1078-0432.CCR-16-3133

26. Hou H, Sun D, Zhang X. The role of MDM2 amplification and overexpression in therapeutic resistance of malignant tumors. Cancer Cell Int. 2019;19:216. doi:10.1186/s12935-019-0937-4

27. Adashek JJ, Kato S, Ferrara R, Lo Russo G, Kurzrock R. Hyperprogression and immune checkpoint inhibitors: hype or progress? [published online ahead of print, 2019 Nov 20]. Oncologist. 2019. doi:10.1634/theoncologist.2019-0636

28. Gao L, Lang L, Zhao X, Shay C, Shull AY, Teng Y. FGF19 amplification reveals an oncogenic dependency upon autocrine FGF19/ FGFR4 signaling in head and neck squamous cell carcinoma. Oncogene. 2019;38(13):2394-2404. doi:10.1038/s41388-018-0591-7

29. Zhang X, Kong M, Zhang Z, et al. FGF19 genetic amplification as a potential therapeutic target in lung squamous cell carcinomas. Thorac Cancer. 2017;8(6):655-665. doi:10.1111/1759-7714.12504

30. Ahn SM, Jang SJ, Shim JH, et al. Genomic portrait of resectable hepatocellular carcinomas: implications of RB1 and FGF19 aberrations for patient stratification. Hepatology. 2014;60(6):1972-1982. doi: $10.1002 /$ hep. 27198

31. Harding JJ, Nandakumar S, Armenia J, et al. Prospective genotyping of hepatocellular carcinoma: clinical implications of next-generation sequencing for matching patients to targeted and immune therapies. Clin Cancer Res. 2019;25(7):2116-2126. doi:10.1158/1078-0432. CCR-18-2293

32. Li F, Li Z, Han Q, et al. Enhanced autocrine FGF19/FGFR4 signaling drives the progression of lung squamous cell carcinoma, which responds to mTOR inhibitor AZD2104. Oncogene. 2020;39 (17):3507-3521. doi:10.1038/s41388-020-1227-2

33. Tan Q, Li F, Wang G, et al. Identification of FGF19 as a prognostic marker and potential driver gene of lung squamous cell carcinomas in Chinese smoking patients. Oncotarget. 2016;7(14):18394-18402. doi:10.18632/oncotarget.7817 
34. Reuben A, Zhang J, Chiou SH, et al. Comprehensive T cell repertoire characterization of non-small cell lung cancer. Nat Commun. 2020;11 (1):603. doi:10.1038/s41467-019-14273-0

35. Reuben A, Gittelman R, Gao J, et al. TCR repertoire intratumor heterogeneity in localized lung adenocarcinomas: an association with predicted neoantigen heterogeneity and postsurgical recurrence. Cancer Discov. 2017;7(10):1088-1097. doi:10.1158/ 2159-8290.CD-17-0256

36. Liu YY, Yang QF, Yang JS, et al. Characteristics and prognostic significance of profiling the peripheral blood T-cell receptor repertoire in patients with advanced lung cancer. Int J Cancer. 2019;145 (5):1423-1431. doi:10.1002/ijc.32145

37. Robert L, Tsoi J, Wang X, et al. CTLA4 blockade broadens the peripheral T-cell receptor repertoire [published correction appears in Clin Cancer Res. 2015 Jul 15;21(14):3359]. Clin Cancer Res. 2014;20(9):2424-2432. doi:10.1158/1078-0432.CCR-13-2648

38. Hogan SA, Courtier A, Cheng PF, et al. Peripheral blood TCR repertoire profiling may facilitate patient stratification for immunotherapy against melanoma. Cancer Immunol Res. 2019;7 (1):77-85. doi:10.1158/2326-6066.CIR-18-0136

39. Britanova OV, Putintseva EV, Shugay M, et al. Age-related decrease in TCR repertoire diversity measured with deep and normalized sequence profiling. $J$ Immunol. 2014;192(6):2689-2698. doi:10.4049/jimmunol.1302064
40. Zhu W, Peng Y, Wang L, et al. Identification of $\alpha$-fetoprotein-specific T-cell receptors for hepatocellular carcinoma immunotherapy. Hepatology. 2018;68(2):574-589. doi:10.1002/hep.29844

41. Heim K, Neumann-Haefelin C, Thimme R, Hofmann M. Heterogeneity of HBV-specific CD8+ T-cell failure: implications for immunotherapy. Front Immunol. 2019;10:2240. doi:10.3389/ fimmu.2019.02240

42. Di Blasi D, Boldanova T, Mori L, Terracciano L, Heim MH, De Libero G. Unique T-cell populations define immune-inflamed hepatocellular carcinoma. Cell Mol Gastroenterol Hepatol. 2020;9 (2):195-218. doi:10.1016/j.jcmgh.2019.08.004

43. Tan AT, Yang N, Lee Krishnamoorthy T, et al. Use of expression profiles of HBV-DNA integrated into genomes of hepatocellular carcinoma cells to select $\mathrm{T}$ cells for immunotherapy. Gastroenterology. 2019;156(6):1862-1876.e9. doi:10.1053/j. gastro.2019.01.251

44. Han JW, Yoon SK. Tissue-resident lymphocytes: implications in immunotherapy for hepatocellular carcinoma. Int $J \mathrm{Mol}$ Sci. 2020;22(1):232. doi:10.3390/ijms22010232

45. El-Khoueiry AB, Kim RD, Harris WP, et al. Updated results of a phase $1 \mathrm{~b}$ study of regorafenib (REG) $80 \mathrm{mg} /$ day or $120 \mathrm{mg}$ /day plus pembrolizumab (PEMBRO) for first-line treatment of advanced hepatocellular carcinoma (HCC). $J$ Clin Oncol. 2021;39 (15_suppl):4078. doi:10.1200/JCO.2021.39.15_suppl.4078
Journal of Hepatocellular Carcinoma

\section{Publish your work in this journal}

The Journal of Hepatocellular Carcinoma is an international, peerreviewed, open access journal that offers a platform for the dissemination and study of clinical, translational and basic research findings in this rapidly developing field. Development in areas including, but not limited to, epidemiology, vaccination, hepatitis therapy, pathology and molecular tumor classification and prognostication are all considered for publication. The manuscript management system is completely online and includes a very quick and fair peer-review system, which is all easy to use. Visit http://www.dovepress.com/ testimonials.php to read real quotes from published authors. 